\title{
PERCEPCIONES SOBRE LAS CONSECUENCIAS SOCIALES DEL ESTADO DEL BIENESTAR Y SU FUTURO
}

\section{PERCEPTIONS ON THE SOCIAL CONSEQUENCES AND THE FUTURE OF WELFARE STATE}

\author{
Francisco García Tortosa \\ Universidad de Alicante \\ fgtortosa@ua.es
}

Recibido: 6/5/2010

Aceptado: 2/6/2010

\begin{abstract}
Resumen
Las actitudes ciudadanas ante el estado del bienestar van a ser en el futuro motivo de gran interés debido a los proyectos de reforma que se prevee realizar. Los valores y los intereses personales son el conjunto de factores que influyen decisivamente en la opinión de los ciudadanos.

Este trabajo pretende dilucidar en que medida los valores representados por la idea de igualdad, de autoridad y de mérito, la posición política y la percepción de los efectos del estado del bienestar permiten determinar la opinión de los individuos sobre la situación futura del sistema de pensiones.
\end{abstract}

Palabras clave: estado del bienestar, actitudes ciudadanas, pensiones, meritocrácia, igualdad, autoridad.

\begin{abstract}
Citizens' attitudes to the welfare state will be in the future of great interest because of the draft reform envisaged. The values and interests personal are all factors that strongly influence in the opinion of citizens.

This paper seeks to ascertain to what extent values represented by the idea of equality, authority and merit, political position and perceptions of the effects of state welfare for determining the views of individuals on the future status of the pension system.
\end{abstract}

Keywords: welfare state, civic attitudes, pensions, meritocracy, equality, authority. 


\section{INTRODUCCIÓN}

Nuestro actual modelo de estado del bienestar y su posible reforma van a aparecer en el futuro como uno de los asuntos más importantes que serán objeto de debate en la sociedad española. Existen graves dificultades para realizar proyecciones de futuro en estos temas y problemas de entendimiento en la población, principalmente en sus mecanismos de funcionamiento. La amenaza de fuertes recortes y las sospechas de intereses ocultos en las decisiones políticas complican todavía más la situación, dificultando un debate imprescindible. El análisis de los mecanismos que construyen la opinión pública sobre el funcionamiento y las consecuencias del estado del bienestar son pues unas de las tareas fundamentales de la sociología para este comienzo de siglo.

A la hora de analizar las políticas públicas del bienestar es fundamental tener presente a qué nos estamos refiriendo. Para el caso que nos ocupa entendemos como políticas del bienestar aquellas que «tienen como objetivo garantizar la equidad y la seguridad socio-económica» (Del Pino 2005:4). Históricamente se sitúa el comienzo de este tipo de políticas en la Alemania de Bismark a finales del siglo XIX con la creación de los subsidios de enfermedad y jubilación, aunque su adopción se generalizo a partir de la II Guerra Mundial, con la adopción por parte de los estados europeos del modelo económico keynesiano.

Los distintos modelos de estado del bienestar europeos o mundos del capitalismo de bienestar según la clasificación de Esping-Andersen se pueden dividir en tres modalidades principales: el capitalismo de bienestar liberalanglosajón, que solo cubre los riesgos de pobreza extrema; el capitalismo del bienestar conservador-corporatista o europeo occidental, que parte del principio de subsidiariedad, donde el estado solo actúa cuando fallan el resto de las redes de apoyo; el capitalismo de bienestar socialdemócrata-nórdico donde el estado cubrió todos los riesgos prestando servicios universales y el llamado "régimen mediterráneo de bienestar" caracterizado por la ausencia de una cobertura especifica del riesgo de exclusión descargando aquel riesgo exclusivamente sobre la familia y la beneficencia (Espina Moreno 2004). Algunos autores entienden que el régimen mediterráneo no es más que un submodelo del capitalismo de bienestar conservador cuyas particularidades están determinadas por su evolución histórica.

Las políticas del bienestar españolas son muy recientes comparadas con las del resto de Europa Occidental. Aunque el establecimiento de un sistema de pensiones y de una cobertura sanitaria universal correspondió a la Dictadura de Franco, el grueso de políticas sociales en su actual configuración comienza con la llegada de la democracia en España a partir de 1978. 
Hoy en día todavía se deja notar en las actitudes y en los valores de los españoles, esta herencia de la dictadura por el paternalismo con que se afrontan estas políticas y por la fuerte influencia de la iglesia católica, que ha ido poco a poco reocupando los espacios públicos de los que fue desalojada especialmente en educación y en la asistencia social.

Dentro del periodo democrático se pueden distinguir claramente tres fases en la evolución del estado del bienestar en España: una que abarca desde los años 80 hasta los primeros 90 , donde se dio un fuerte crecimiento del gasto público y de la cantidad y calidad de los servicios, otro que llega hasta el final del siglo donde se produce una fuerte contención del gasto y una disminución o vuelta atrás de determinados servicios, incorporando a la empresa privada en la prestación de algunos de estos servicios, especialmente en lo que se refiere a la sanidad y a los servicios sociales y por ultimo un tercero donde se vuelven a potenciar determinadas prestaciones y se incorporan otras más cercanas al modelo del bienestar socialdemócrata (Del Pino 2005).

Las actitudes ante el estado del bienestar son favorables en la mayoría de los países europeos. Solo determinados grupos sociales (principalmente aquellos situados en un estatus más alto) ponen el énfasis en un cambio del modelo de gasto en función del interés propio (Noya Miranda 1999). En España, que no es una excepción en este tema, existe un fuerte apoyo a las políticas públicas del bienestar, especialmente a las pensiones y al sistema sanitario. Ambas instituciones suscitan la mayor tasa de apoyo en la ciudadanía, inclinándose la mayoría de la población por un modelo estatalista-universalista (entre un 60\% y un $70 \%$ de la población) con un pequeño porcentaje cada vez más numeroso que está a favor de un modelo residualista en el que el estado sea responsable únicamente de los más desfavorecidos (Del Pino 2007).

Este mayoritario apoyo del sistema del bienestar clásico europeo sostenido por aportaciones individuales y gestionado por el estado se justifica por la percepción de que nuestro sistema del bienestar no está completo, que hay zonas oscuras que no han sido todavía cubiertas por las prestaciones del estado que se continua apoyando en las familias (principalmente en la mujer) para suplir estos vacíos. La percepción de una fuerte desigualdad no superada por el periodo democrático es otra fuente de apoyo a nuestro sistema del bienestar, especialmente por lo grupos más desfavorecidos por el actual modelo económico (mujeres y jóvenes).

Uno de los problemas más importantes del estado del bienestar en España es que buena parte de los ciudadanos no llega a comprender sus mecanismos del funcionamiento, lo que se traduce en unas expectativas y unos requerimientos excesivos. Diferentes autores han ligado este exceso de demandas 
con el bajo nivel cognitivo que muestran los ciudadanos hacia la política y consecuentemente con su capacidad para comprender sus mecanismos. Hay que tener en cuenta la extrema juventud de nuestro sistema democrático (respecto al resto de los países europeos) y que este basó su legitimidad en la construcción de un estado del bienestar homologable a los europeos. Cualquier modificación o reforma que se quiera realizar deberá contar con las dificultades que tiene la población "para aceptar el hecho mismo de que sus instituciones hayan entrado en crisis nada mas ser instauradas" (Pradera 1995:42) Un interesante ejemplo de esto han sido las reacciones que se han dado a partir de la propuesta del gobierno socialista de aumentar la edad de jubilación hasta los sesenta y siete años y como el conjunto de actores sociales relevantes han respondido a esta propuesta.

El fuerte apoyo al estado del bienestar está en España muy relacionado con el importante sentimiento de igualdad que aparece aquí como un rasgo distintivo frente al resto de los países de Europa Occidental y que compartimos con los países excomunistas. Estamos pues en una sociedad mayoritariamente igualitaria y estatista (Noya Miranda 1999:186). A la hora de analizar a un determinado país hay que tener presente cuáles son los valores dominantes, aquellos que aparecen como hegemónicos. En nuestro caso el valor dominante es la igualdad y el valor conflictivo es el individualismo lo que va a dar lugar a una mayor ambivalencia entre los defensores del mérito frente a los defensores de la igualdad a la hora de hablar de su respaldo a las políticas del bienestar y a sus consecuencias.

Otra de las dimensiones especialmente significativas a la hora de analizar las actitudes de los españoles es la de la dimensión autoridad-tolerancia. La sociedad ha ido cambiando mayoritariamente, desde el comienzo de la transición, pasando de unos valores de permisividad/tolerancia hacia una preferencia por la autoridad. En este sentido se deja notar la influencia de cincuenta años de dictadura, tanto en la posición inicial de absoluto rechazo de la autoridad como en su actual auge que da cuenta de un fuerte neofranquismo. Así, en las últimas encuestas sobre valores se observa que los jóvenes (que en su mayoría defienden el sistema democrático como el mejor posible) valoran como opción un gobierno autoritario en un alto porcentaje (Elzo 2000).

El español se declara mayoritariamente poco interesado por la política y consecuentemente muestra una escasa capacidad para analizar cuestiones que tengan que ver con fenómenos políticos. La población se ha autoposicionado sistemáticamente desde los años 80 en el centro izquierda (que corresponde a las posiciones 3,4 y 5 de la escala) rasgo que compartimos con nuestros vecinos griegos e italianos. Ahora bien, existen fuertes evidencias de que 
la invariabilidad de este posicionamiento en realidad esconde un cambio de valores de manera que lo que suponía a principio de la transición situarse en el centro-izquierda es muy distinto a lo que supone hoy en día, existiendo un fuerte deslizamiento hacia el autoritarismo (Alaminos 2004).

Por otro lado hay que destacar las dificultades de muchas personas para autoubicarse correctamente, lo que estaría en consonancia con la falta de conocimientos políticos citada anteriormente. En este sentido se ha propuesto la utilización de la dimensión "igualdad-desigualdad" para sortear estas dificultades. Para Bobbio, la igualdad es el rasgo clave para entender la diferencia entre la izquierda y la derecha. Este factor es complementado por la dimensión "autoritarismo-tolerancia" que permite graduar o matizar a la dimensión igualitaria, de tal forma que la igualdad permite graduar las posiciones de izquierda mientras que la autoridad lo hace con las de derecha (Alaminos 2004).

La simplicidad a la hora de responder al tipo de preguntas que componen estos factores y su baja tasa de no respuesta juega a favor de este sistema frente al clásico autoposicionamiento. En este trabajo se ha optado por utilizar ambos sistemas ya que se entiende que pueden funcionar de manera complementaria para evitar problemas de validez en la investigación y porque aportan una mayor riqueza a la explicación.

La cultura nacional del estado del bienestar (que apoya sin reservas el modelo igualitarista y estatalista) ha estado muy influida por las circunstancias políticas y sociales que propiciaron su implantación. Por un lado tenemos la influencia del ya existente modelo de pensiones y asistencia sanitaria, con sus conocidos problemas de cobertura y recursos. Por otro, la influencia del modelo continental de estado del bienestar, ya comentado en anteriores lineas. Con todo ello, a partir de los Pactos de la Moncloa se produce un acuerdo entre el conjunto de las fuerzas políticas democráticas cuyas consecuencias son la implantación de un modelo de estado del bienestar caracterizado por su carácter contributivo y dual, ampliamente sesgado a favor de un determinado colectivo (hombres, con trabajo estable y mayores). Inicialmente estas políticas de bienestar son fuertemente apoyadas por el conjunto de la población.

Con el paso del tiempo el modelo entra en un estado de casi quiebra, a partir del cual comienzan a cobrar importancia las políticas del estado del bienestar en las correspondientes campañas electorales. En un intento de reforzar la situación del sistema de pensiones y de impedir la huida de la clase media hacia planes privados, se firma el Pacto de Toledo en el que se acuerda por parte de todos los grupos políticos, los sindicatos mayoritarios y la patronal alejar del debate público la situación de la seguridad social y sus posibles reformas. A partir de este momento, la opinión pública ha estado recibiendo 
solo mensajes tranquilizadores sobre la situación del estado del bienestar y se ha imposibilitado de facto la discusión sobre los problemas y las soluciones que necesariamente sin un pacto de silencio se hubiera dado. Esto se hace manifiesto en el optimismo con el que se ve la situación futura del sistema de pensiones en España frente a las opiniones bastante menos optimistas del resto de los países de nuestro entorno.

Para Del Pino "las actitudes ciudadanas hacia el bienestar se explican en función de dos conjuntos de factores: a) los valores y b) los intereses, determinado por el grado de dependencia personal de los programas sociales". (Del Pino 2007:187). El problema de la determinación de la influencia de unos $\mathrm{u}$ otros factores, que podríamos situar en el nivel macro y nivel micro respectivamente ha sido discutido intensamente por Hans-Jürgen Andreß y Thorsten Heien que contemplan un conjunto de cuatro factores separando los intereses en dos factores distintos: a) el interés propio, b) los valores y las normas, c) los diferentes patrones de socialización y d) las culturas nacionales del estado del bienestar (Andre $\beta$ y Heinen 1999) Este modelo multinivel tiene mucho que ver con el tipo de análisis realizado en la República Federal Alemana a partir de la reunificación, donde es muy importante el peso de las distintas culturas nacionales del estado del bienestar (occidental y oriental), de la misma forma que si el análisis se efectuará sobre países distintos.

El problema del modelo propuesto por Andreß y Heien estriba en la dificultad para separar el interés propio de los patrones de socialización. Por ejemplo como es sabido existen diferentes patrones de socialización entre hombres y mujeres, de tal manera que las mujeres suelen puntuar más positivamente los valores que inciden en la igualdad mientras que los hombres lo hacen sobre aquellos que inciden en el merito. Al mismo tiempo también es habitual que sean las mujeres las encargadas del cuidado de niños y ancianos. De esta forma cuando las mujeres muestran sus preferencias por un estado del bienestar que profundice en la igualdad de oportunidades, ien que medida se debe al interés propio o a sus diferentes patrones de socialización?

La dificultad para decidir cuales son los factores determinantes estriba también en que no siempre unas características sociodemográficas posicionan a los individuos en un determinado lugar dado que

las identidades y los intereses de los actores sociales no están predeterminados desde sus posiciones estructurales. Se crean en un proceso donde el marco institucional en el que la gente actúa, y las tradiciones históricas a través de las cuales los acontecimientos y los procesos se interpretan, tienen un impacto decisivo. El peso de la exposición a diferentes regímenes institucionales crea divergentes visiones del mundo, incluso entre las personas en similares posiciones estructurales (Svallfors 1997: 291) citado por (Andre y Heien 1999). 


\section{MODELO CAUSAL}

Para la realización de nuestro modelo casual nos centraremos en la dimensión macro del problema utilizando como variables independientes las dimensiones igualdad/desigualdad, autoridad/tolerancia y mérito. Estas dimensiones se complementan en un segundo nivel explicativo con el autoposicionamiento político, con las opiniones ante las consecuencias socialmente positivas del estado del bienestar, en particular respecto a la reducción de la pobreza y a la igualación del nivel de renta entre individuos y con las opiniones sobre las consecuencias económicamente negativas del mantenimiento de estas políticas, en particular del coste que tiene para las empresas y para la competitividad del país.

Nuestra hipótesis es que la igualdad junto con la autoridad determinan la posición política de los individuos y al mismo tiempo están relacionadas con la percepción de los efectos positivos y negativos del estado del bienestar. Un alto valor de igualdad se corresponde con un autoposicionamiento más a la izquierda y con una opinión favorable de los efectos igualitarios del estado del bienestar. De la misma forma un alto valor de autoridad se corresponde con un autoposicionamiento más hacia la derecha y con una opinión desfavorable sobre los consecuencias económicas que tiene sobre las empresas el estado del bienestar.

\section{SISTEMA DE BIENESTAR ESPAÑOL}

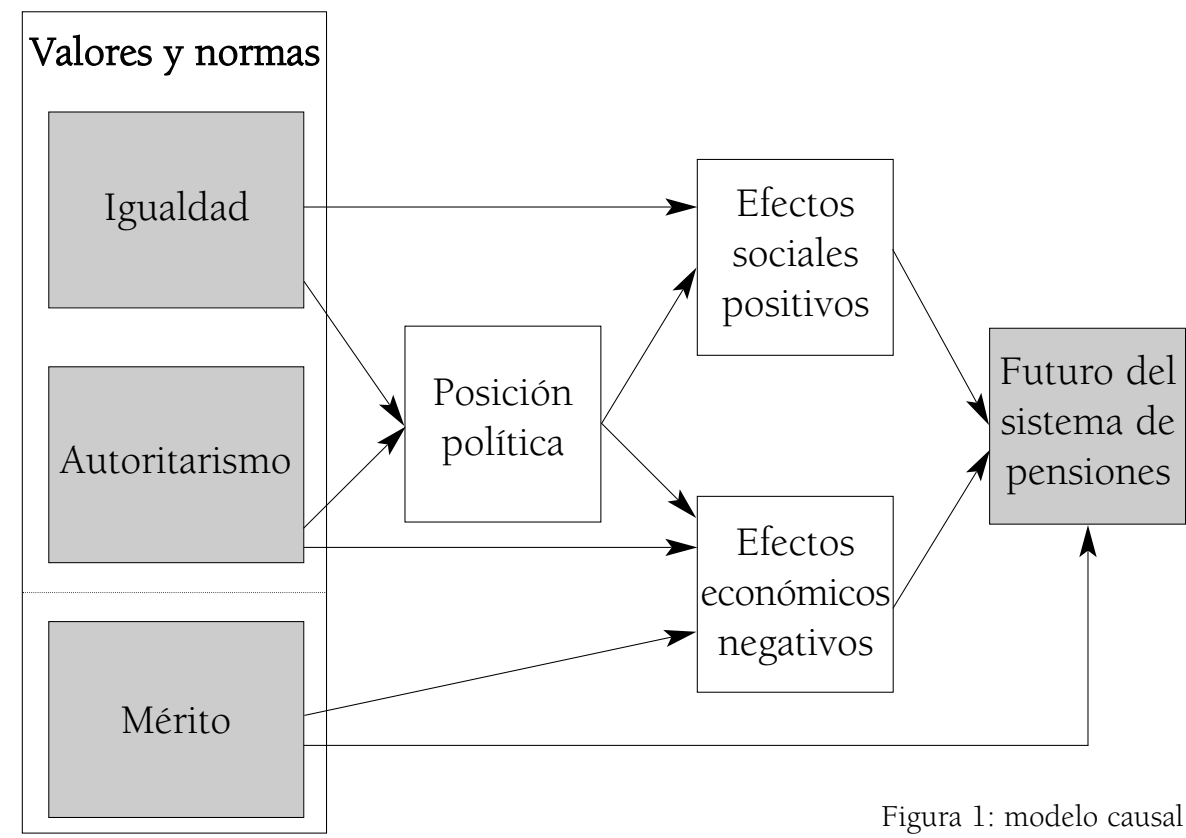


Ambas percepciones sobre los efectos económicos negativos en las empresas y sobre los efectos positivos para la justicia social del estado del bienestar tendrán consecuencias en la previsión sobre el futuro de las pensiones.

El papel que juega el mérito como valor en la sociedad es más contradictorio. La hipótesis del papel del merito en el modelo es que juega un papel ambivalente (Noya Miranda 1999), justificando la función del estado del bienestar y al mismo tiempo cuestionándolo, en virtud de sus efectos económicos perjudiciales. En cuanto a su papel en el autoposicionamiento político es poco relevante a la hora de justificar el voto, no habiéndose configurado a día de hoy como una alternativa ni a la igualdad y ni a la autoridad, existiendo individuos con posiciones meritocráticas en ambos colectivos. Por lo tanto el mérito es más una representación simbólica de la dimensión que mide el auto interés y/o los diferentes patrones de socialización de los individuos (es decir, su dimensión microsociológica). Al no ser una valor dominante en nuestra sociedad no es capaz de aglutinar movimientos políticos a su alrededor.

\section{METODOLOGÍA}

La investigación va a utilizar como datos secundarios los de la ESE (Encuesta Social Europea) en su cuarta edición (2008-2009) utilizándose únicamente el fichero de datos de España que posee un total de 2576 casos.

Como variables independientes tomaremos los factores igualdad y autoridad que han sido calculados utilizando cada uno de ellos dos variables del cuestionario. El valor igualdad ha sido calculado a partir de dos variables: aquella que sitúa a una sociedad como justa cuando las diferencias entre los niveles de vida de la gente son pequeñas y aquella que responsabiliza al gobierno de la reducción de las diferencias sociales y el factor autoridad a partir de dos variables: la que indica la necesidad de que en los colegios se enseñe a los niños a obedecer la autoridad y la que pide un aumento de las penas para los delitos. El mérito se ha calculado a partir de la variable que indica como aceptable las grandes diferencias de ingresos entre la gente en función de sus diferentes habilidades y esfuerzos.

Como variables dependientes tomaremos el autoposicionamiento político, que mide la posición política del encuestado en una escala desde el 0 (izquierda) hasta el 10 (derecha). Esta variable ha sido transformada reemplazando los casos perdidos con el valor de la media. Para ello se ha tenido en cuenta que en el centro izquierda (que es el valor medio del autoposicionamiento) está la mayoría de los que no saben o no contestan (Elzo 2000).

El factor que mide los efectos económicos negativos del estado del bienestar ha sido calculado utilizando las puntuaciones factoriales de las variables 
que miden el acuerdo o desacuerdo de la gente sobre la afirmación de que las prestaciones y los servicios sociales en España ejercen demasiada tensión en la economía y un coste demasiado elevado para las empresas. Para los efectos sociales positivos se ha utilizado igualmente el análisis factorial utilizando las variables que miden en que medida las prestaciones y servicios sociales en España evitan que se extienda la pobreza y contribuyen a una sociedad más igualitaria.

La variable sobre el coste futuro del sistema de pensiones solicita la opinión de los entrevistados sobre la situación de este dentro de diez años en cuanto a si se podrán mantener o aumentar las prestaciones actuales o bien se tendrán que limitar.

Las variables utilizadas en la investigación son las siguientes:

- Igualdad: Factor que mide el nivel de igualdad. El factor se calcula a partir de las puntuaciones de las siguientes variables:

- gincdif: Para que una sociedad sea justa las diferencias entre los niveles de vida de la gente deberían ser pequeñas

- smdfslv: El gobierno debería tomar medidas para reducir las diferencias en los niveles de ingresos

- Autorida: Factor que mide el nivel de autoridad. El factor se calcula a partir de las puntuaciones de las siguientes variables:

- schtaut: En los colegios se debería enseñar a los niños a obedecer a la autoridad

- hrshsnt: La gente que viola la ley debería ser condenada de forma mucho más dura que ahora

- Merito: Variable que mide el nivel meritocrático. La variable representa a la siguiente pregunta del cuestionario:

- dfincac: Que haya grandes diferencias de ingresos entre la gente es algo aceptable ya que así se premian de forma adecuada las diferentes habilidades y esfuerzos de cada uno

- Aupospol: Auto posicionamiento político. La variable representa a la siguiente pregunta del cuestionario:

- Irscale: En política a veces se habla de "izquierda" y "derecha". Utilizando esta tarjeta, ¿dónde se colocaría usted en esta escala?. El 0 quiere decir izquierda y el 10 derecha.

- Efecsoc: Efectos sociales positivos del estado del bienestar. El factor se calcula a partir de las puntuaciones de las siguientes variables:

- sbprvpv: En qué medida está usted de acuerdo o en desacuerdo con que las prestaciones y servicios sociales en España evitan que se extienda la pobreza 
- sbeqsoc: En qué medida está usted de acuerdo o en desacuerdo con que las prestaciones y servicios sociales en España contribuyen a una sociedad más igualitaria

- Efecec: Efectos económicos negativos del estado del bienestar. El factor se calcula a partir de las puntuaciones de las siguientes variables:

- sbstrec: En qué medida está usted de acuerdo o en desacuerdo con que las prestaciones y servicios sociales en España ejercen demasiada tensión en la economía del país

- sbbsntx: En qué medida está usted de acuerdo o en desacuerdo con que las prestaciones y servicios sociales en España suponen un coste demasiado elevado para las empresas, que tienen que pagar más impuestos y más cargas

- Situpen: Opinión sobre el estado del sistema de pensiones dentro de diez años. La variable representa a la siguiente pregunta del cuestionario, que ha sido transformada en una escala del -1 (España no se podrá permitir unas pensiones como las actuales) hasta 1 (España se podrá permitir subir las pensiones de jubilación)

- lvoapen: Hoy en día también se debate sobre el coste de las pensiones en España. Pensando en cómo será la situación dentro de 10 años, ¿cuál de las frases que aparecen en esta tarjeta se acerca más a su propia opinión? Dentro de diez años: 1. España no se podrá permitir unas pensiones de jubilación como las actuales. 2. España se podrá seguir permitiendo unas pensiones de jubilación como las actuales pero no podrá subirlas. 3. España se podrá permitir subir las pensiones de jubilación. 8 . No sabe (no sugerir)

\section{RESULTADOS}

La matriz de efectos del modelo propuesto es la siguiente:

\begin{tabular}{l|c|c|c|c|c|c|c}
\hline & Aupospol & Efecsoc & Efecec & Situpen & Igualdad & Autoridad & Merito \\
\hline Aupospol & - & 0 & 0 & 0 & 1 & 1 & 0 \\
\hline Efecsoc & 0 & - & 0 & 0 & 1 & 0 & 0 \\
\hline Efecec & 1 & 0 & - & 0 & 0 & 1 & 1 \\
\hline Situpen & 1 & 1 & 1 & - & 0 & 0 & 1 \\
\hline
\end{tabular}


Según estas relaciones, el modelo presenta una densidad de 0,41666.

A continuación se presentan las ecuaciones estructurales correspondientes, donde se muestran los valores estimados para cada relación

$$
\text { situpen }=-0.024^{*} \text { aupospol }-0.075^{*} \text { efecec }+0.087^{*} \text { efecsoc }+0.053^{*} \text { merito }
$$

La opinión sobre situación de las pensiones dentro de 10 años esta determinada negativamente por el autoposicionamiento político (.024), es decir, que cuanto más a la derecha se sitúa el individuo peor es su opinión, negativamente por aquellos que piensan que el estado del bienestar tiene efectos económicos perjudiciales (.075), positivamente por aquellos que tienen una opinión sobre los efectos sociales beneficiosos del estado del bienestar (.087) y por aquellos que defienden el mérito como un buen indicador de las diferencias salariales (.053). Las relaciones son significativas con unos t-value de $-2.66,-4.43,5.21$ y 3.33 respectivamente.

$$
\text { aupospol }=0.31 * \text { autoridad }-0.29 * \text { igualdad }
$$

El autoposicionamiento político depende positivamente del valor del factor de autoridad (.31), es decir, los más autoritarios se sitúan más hacia la derecha, y negativamente del valor del factor de igualdad (.29), es decir, los más igualitarios se sitúan más hacia la izquierda. Ambas relaciones son significativas con unos t-value de 7.30 y -7.00 respectivamente.

$$
\text { efecec }=0.056 * \text { aupospol }+0.14 * \text { merito }+0.14 * \text { autoridad }
$$

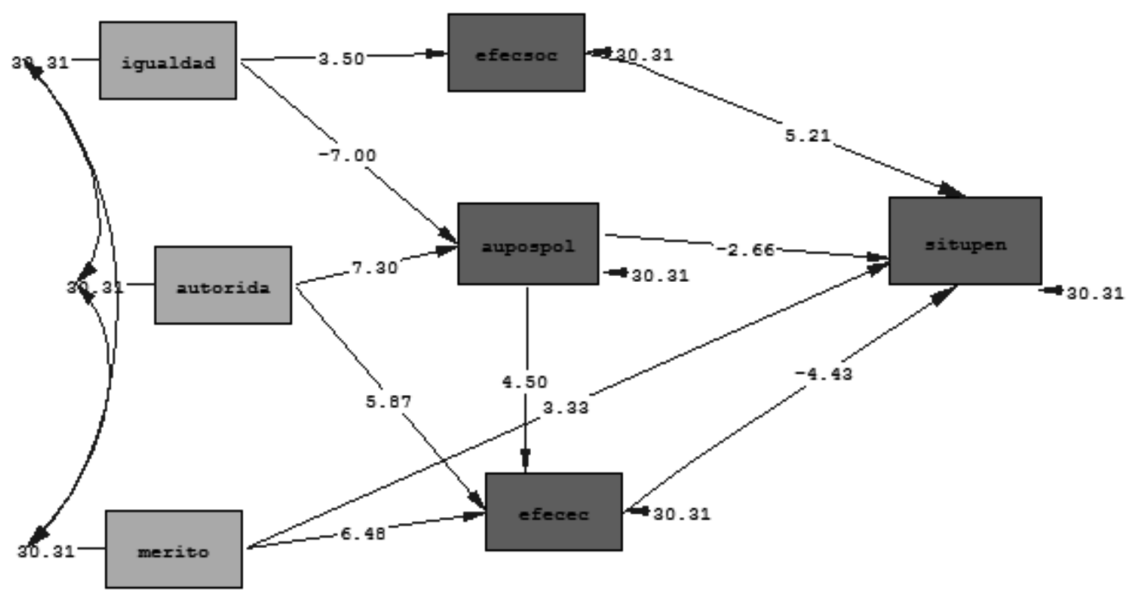

Figura 2: modelo causal con efectos

Chi-Square $=7.78, \mathrm{df}=8, \mathrm{P}-\mathrm{value}=0.45485, \mathrm{RMSEA}=0.000$ 
La opinión sobre los efectos económicos negativos del estado del bienestar aumenta cuanto mayor sea el autoposicionamiento político (.056), es decir, mayor en los individuos más hacia la derecha, mayor entre los meritocraticos (.14) y entre los que defienden valores autoritarios (.14). Las relaciones son significativas con unos t-value de $4.50,6.48$ y 5.87 respectivamente.

$$
\text { efecsoc }=0.080 * \text { igualdad }
$$

Por último, la opinión sobre los efectos sociales positivos del estado del bienestar es mayor cuanto mayor sea la posición igualitaria del individuo (.080). La relación es significativa con un t-value de 3.50.

Tal y como se puede observar en los t-values de la figura 2 todas las relaciones son significativas con valores de $\mathrm{T}$ superiores a $|2|$. Los estadísticos de ajuste (en la tabla siguiente) indican un buen ajuste del modelo

\begin{tabular}{l|c|c|c}
\hline Estadístico & Variación & Valores recomendables & Valores del modelo \\
\hline RMSEA & & $<0.05$ & 0.00 \\
\hline P-Value & $0-1$ & $>0.05$ & 0.45485 \\
\hline GFI & $0-1$ & $>0.90$ & 1 \\
\hline AGFI & $0-1$ & $>0.90$ & 1 \\
\hline NFI & $0-1$ & $>0.80$ & 0.98 \\
\hline NNFI & $0-1$ & $>0.95$ & 0.98 \\
\hline
\end{tabular}

\section{DISCUSIÓN Y CONCLUSIONES}

Las percepciones sobre el estado del bienestar son muy complejas de medir. Existen múltiples determinantes sobre las opiniones individuales, siendo en principio las más importantes los valores y los intereses individuales.

Las posiciones políticas de los españoles están determinadas por dos valores fundamentales en nuestra sociedad: la igualdad y la autoridad. El autoposicionamiento político dependerá del mayor o menor énfasis en estos valores, donde la igualdad reforzara las posiciones de izquierda mientras que la autoridad los hará sobre las de derechas. Aunque las posiciones políticas declaradas no han variado en 20 años no parecen decir lo mismo que al principio de la democracia ya que cada vez tiene más valor en la sociedad el autoritarismo frente a la tolerancia lo que supone una derechización de facto. En este sentido no hay que descartar la importancia del envejecimiento de la población para entender el viraje ideológico. 
Las posiciones sobre nuestro estado del bienestar están determinadas por la posición política (en menor medida) y principalmente por la percepción de sus efectos positivos y/o negativos. Dado que la percepción positiva viene determinada por la igualdad y la negativa por la autoridad en la medida que varíen ambas posiciones cambiara la opinión sobre el futuro del sistema de pensiones, que en estos momentos es mayoritariamente optimista. Es evidente que el futuro envejecimiento de la población influirá en una mayor valoración del sistema de pensiones independientemente de la posición política de los individuos.

La importancia de las posiciones meritocráticas en nuestra sociedad es limitada en tanto que ideológicamente no es un valor dominante, por lo que, los que defienden este valor tienen posiciones ambivalentes apoyando el sistema de pensiones y al mismo tiempo afirmando las consecuencias negativas de este. La evolución histórica de este valor ha sido distinta de lo esperado, ya que durante el primer periodo democrático fue aumentado para volver a posiciones iniciales en los años 90. Esto contradice las teorías defendidas por los estudiosos del cambio de valores postmaterialistas, que entendían que se iba a producir una convergencia con el resto de Europa. Sin embargo, la percepción social de la existencia de fuertes desigualdades no modificadas significativamente en el periodo democrático y los problemas de dualización social (fundamentalmente en el mercado de trabajo) son coherentes con este cambio de posición con la vuelta de posiciones igualitarias en detrimento de las meritocráticas.

El modelo propuesto es probablemente demasiado simple para dar cuenta de la complejidad de relaciones que se dan en una sociedad moderna a la hora de valorar el estado del bienestar. La importancia del interés propio es evidente y en este modelo solo se ha trabajado con las variables que miden valores. Además estas variables al ser ordinales tienen importantes problemas de validez para su utilización en aplicaciones como LISREL. Por otro lado un modelo a partir de la técnica MIMIC sería más realista al estar menos compartimentado, aceptando que existen valores comunes en una sociedad y que se pueden defender al mismo tiempo posiciones igualitarias, meritocráticas y autoritarias sin caer en demasiadas contradicciones.

\section{BIBLIOGRAFÍA}

ALAMINOS, A. (2004). "Tendencias en ideología política: estructura y contenidos." en Tendencias en identidades, valores y creencias, Colección de ciencias sociales. Madrid: Editorial Sistema.

ALAMINOS, A, FRANCÉS, F. y SANTACREU, O. (2005). Socialización, ideología y participación. Casos prácticos de análisis estructural de covarianzas. San Vicente del Raspeig: Observatorio Europeo de Tendencias Sociales. 
ANDREB, HJ., y HEIEN, T. (1999). "Four Worlds of Welfare State Attitudes? A comparison of Germany, Norway, and The United States." http://eswf.uni-koeln.de/ forschung/wme/WME_AP8.pdf. (Accedido Marzo 26, 2010).

ANDRÉS ORIZO, F. (1996). Sistemas de valores en la España de los 90. $1^{\circ}$ ed. Madrid: Centro de Investigaciones Sociológicas; Siglo Veintiuno de España Editores.

BENEDICTO, J. (1993). “ ¿Espectadores o actores potenciales?: el debate sobre los sistemas de creencias políticas de los ciudadanos." Revista de Estudios políticos (Nueva Época) 80:271-295.

DEL PINO, E. (2007). "Las actitudes de los españoles hacia la reforma del Estado de Bienestar." Política y Sociedad 44:185-208. http://revistas.ucm.es/cps/11308001/ articulos/POSO0707230185A.PDF. (Accedido Marzo 17, 2010).

DEL PINO, E. (2005). " $¿ S e$ han modificado las preferencias de los ciudadanos sobre las políticas de bienestar en España (1985-2005)?." http://www.ipp.csic.es/doctrab2/dt-0503.pdf. (Accedido Marzo 17, 2010).

ELZO, J. (2000). España 2000, entre el localismo y la globalidad : la encuesta europea de valores en su tercera aplicación, 1981-1999. [Madrid] ;[Bilbao]: Ediciones SM; Universidad de Deusto.

ESPINA MORENO, A. (2004). "Un problema de elección social: la reforma del estado de bienestar en Europa." http://www.realinstitutoelcano.org/documentos/ 114/114.pdf. (Accedido Marzo 17, 2010).

ESPINA MORENO, A. (2004). "Estado del bienestar y teorema de la imposibilidad." ICE 815:61-80.

HEIEN, T., y HOFACKER, D. (1999). "How do welfare regimes influence attitudes? A comparison of five European countries and the United States 1985-1996." http://eswf.uni-koeln.de/forschung/wme/wme_ap9.pdf. (Accedido Marzo 27, 2010).

NOYA MIRANDA, FJ. (1999). "El valor de la ambivalencia: las actitudes ante la meritocrácia, la igualdad y el Estado de Bienestar en España en perspectiva comparada." REIS, vol. 86/99, págs. 185-221.

PRADERA, J. (1995). "El doble lamento. La opinión pública ante el Estado del Bienestar." Claves de razón práctica.

TEZANOS, J, (coord) (2004). Tendencias en identidades, valores y creencias. Madrid: Editorial Sistema. 